\title{
Taking Care of the Staff We Take Care of the Patients: Project for the Humanization of an Acute Geriatric Care Unit
}

\author{
Ruben Octavio Luzardo Batista* \\ Department of Nursing, Universidad de Las Palmas de Gran Canaria, Spain \\ *Corresponding author: Ruben Octavio Luzardo Batista, Department of Nursing, Universidad de Las Palmas de Gran \\ Canaria, Spain
}

\begin{tabular}{|c|c|}
\hline ARTICLE INFO & ABSTRACT \\
\hline $\begin{array}{l}\text { Received: 慧 May 02, } 2021 \\
\text { Published: 幽 May 13, } 2021\end{array}$ & $\begin{array}{l}\text { Citation: Ruben Octavio Luzardo Batista. Taking Care of the Staff We Take Care of the } \\
\text { Patients: Project for the Humanization of an Acute Geriatric Care Unit. Biomed J Sci \& } \\
\text { Tech Res 35(5)-2021. BJSTR. MS.ID.005754. }\end{array}$ \\
\hline
\end{tabular}

\section{Mini Review}

The need for humanization arises today in many areas, both in professional life and in personal relationships, and not only in the field of health, but in many other professions. But it is perhaps in healthcare that the most delicate situation arises, since vulnerability and suffering are almost inherent to the process of becoming ill. The changes in healthcare in recent years have generated numerous debates related to humanization in the care of the sick person. The term humanizing is sometimes controversial, even though it is an intrinsic concept in the health professions. Strictly speaking, "humanize", according to the RAE means: to make someone or something human, familiar and affable. In another meaning, it also means "to soften, soften, become benign" (RAE). Humanization in healthcare is not an ideology, it is an alliance with the person and his or her way of life. It includes aspects of quality and constitutes an ethical commitment to consider the person as a whole: both in terms of autonomy and in its more subjective dimension. To humanize healthcare, it is necessary to respect the fact that each person is unique, unrepeatable, and responds differently to life crises. It is therefore necessary to encompass many areas: assistance (care and caregiver), ethics, culture, professional competencies (communication, skills) and, of course, human resources.

Humanizing has to do with the environment (spaces, well-being, warmth), with interventions of competence and collaboration, with integration into the community, with the individual and the community. All this implies the need for the inclusion of human sciences in the training of healthcare personnel, and, of course, implies protecting the right to maintain control over one's own life. (LÓPEZ et al., 2018). Dehumanization, on the contrary, means depersonalization, and puts the pathological process as the only target of health action. Some of the main causes of dehumanization are: the "dictatorship of technology" that leads to the "reification of the patient", super-specialization, the application of mercantilist criteria to health management, and the denial that suffering and death affect patients, relatives and professionals equally [1].

\section{Justification}

With the use of new technologies in healthcare, the basic and human needs of healthcare professionals, patients themselves and their families have been left in the background. In the hospital setting, nurses assist, from our professional role of caring for users and families, in the different stages of their life cycle. That is why, once the elderly is admitted to an Acute Geriatric Care Unit, they are often subjected to strict rules and schedules and to a more and more technified medical care, from the sophistication and strict organization, which is why the need to combine and accompany the incorporation of technological and scientific advances with the best human values arises. In the field of Geriatrics and in the care of the elderly, crucial moments in the life of every person are experienced, 
so that comprehensive and holistic nursing care of high quality becomes of paramount importance. In this context, after an initial reflection, it is proposed to develop a humanization project in the Acute Geriatric Unit Care of the Hospital Insular de Lanzarote, the project "Humanizing the attention and care of the elderly". It arises as an ambitious project, which tries to engage all the professionals of the Unit, both medical and nursing staff and auxiliary staff and orderlies, with an exceptional involvement and commitment. It is an initiative of solidarity, integration and closeness to patients and relatives, with the idea of aligning ourselves with them, with their needs and expectations, opening our unit, and managing to combine the purely care activity with a much more personalized attention $[2,3]$.

\section{Objectives}

\section{General Objective}

1. To improve the satisfaction of the users and relatives of the Geriatrics Service of the Hospital Insular de Lanzarote.

2. To work to improve the work carried out in the Acute Geriatric Care Unit.

3. To have a greater presence in the community.

\section{Specific Objectives}

1. To make known the specialty of Geriatrics and Geriatric Nursing and the work that is done in the Geriatric Service of the Hospital Insular de Lanzarote, specifically in the Acute Geriatric Unit Care.

2. To improve communication, not only among the professionals of the unit but also with the rest of the professionals of the center and the rest of the units of the Lanzarote Health Area, as well as well as with our patients and families.

3. Encourage motivation at work, looking for tools to care for professionals.

\section{Lines of Action (3 Lines of Action are Proposed)}

\section{Line1 Culture of Humanization in the Professionals of the Geriatrics Service}

General Objectives: The aim will be to extend the culture of humanization to the whole Geriatrics Service including all staff (both healthcare and non-healthcare).

\section{Specific Objectives:}

1. To sensitize all the staff in humanization in the Hospital de Insular de Lanzarote (Geriatrics Service), specifically in the Geriatric Acute Unit.

2. To train all staff in humanization in the Geriatric Service.
3. To enhance teamwork to improve humanization in the team.

\section{General Actions}

\section{Training in Humanization: Content}

1. Concept of humanization.

2. Ethics training

3. Training in confidentiality.

4. Training in interpersonal skills.

5. Communication training: how to communicate bad news, etc.

\section{Training Format}

1. Face-to-face.

2. Blended learning.

3. On line.

\section{General Actions}

1. Humanization standards.

2. Performing a protocol of how to enter a user's cubicle.

3. Draw up a decalogue with the rights of patients/users

\section{Specific Actions}

1. Detection of a group of professionals with a special concern for humanization issues, through informal conversations with the

2. Detection of a group of professionals with special concern for humanization issues, through informal conversations among the team.

3. Organization of work meetings with the members of the Geriatric Acute Unit.

4. Designate a leader, a person who stands out for his or her sensitivity, communication skills, empathy with the team, and interest in the development of the project, who will be in charge of distributing tasks and supervising all the work groups.

5. With the group's contributions and the leader's initiatives, elaborate a work plan and a schedule to be followed.

6. To elaborate a voluntary list to collaborate.

7. Assign each commitment to at least 2 people in charge, from different levels, depending on the topic to be worked on.

8. The intermediate positions (unit managementsupervision) will be in charge of the bureaucratic issues, connection with the bureaucratic issues, liaison with the 
Hospital's management team, external contacts (city councils, media...) and overall coordination.

9. Present the project to the Hospital's management team, and after its approval, initiate internal and external dissemination (posters, press releases, a monthly program on local radio and some on regional channels and television reports, etc.).

\section{Line 2 Taking Care of the Professional, We Take Care of the Patients: Recovering the Vocation}

General Objectives: Working in the field of Geriatrics can be particularly stressful. According to some studies, the rates of "burnout syndrome" can reach up to 50\% in professionals working in geriatrics. The classic symptoms are fatigue, depersonalization and loss of self-esteem. There are risk factors such as work overload, low recognition, continuous contact with life-threatening situations and end-of-life events. It can lead to fatigue, anxiety and depression. The care of the professional is proposed as a necessary measure to guarantee the quality of the care task, seeking motivation and the recovery of vocation in many cases. Another of the objectives is to become aware of the problems of communication with families and among professionals, and to train ourselves in communication tools. Numerous studies have highlighted this deficiency. The acquisition and training of communication skills should be mandatory in the training of healthcare professionals. Interventions along these lines, such as the training of health science students, have led to an improvement. Not only communication with relatives should be improved, but also among the professionals themselves, since lack of communication is responsible for a high percentage of errors.

\section{General Actions}

Communication Training: Provide training in communication skills sessions, decision making in the limitation of life-sustaining limitation of life support therapies, coaching sessions, motivational sessions for professionals carried out by specialist psychologists.

Specific Actions: Show techniques for relaxation such as reiki and cranio-chakral therapy.

Monthly Team Meetings: In this monthly commitment, participate in a day, focused on "self-counseling" or counseling" or the set of skills necessary for the relationship with oneself and others to improve the physical, emotional and spiritual health of the physical, emotional and spiritual health of professionals, and become aware of the need to acquire skills or strategies for self-help and personal growth. In short, emotional, social and motivational gymnastics.

\section{Line 3. Respect for the Privacy of the Elderly Admitted to the Acute Geriatric Care Unit}

General Actions: Detail strategies to jealously protect the privacy of our patients.
Specific Actions: Elaborate a triptych of good practices to guarantee the privacy of the elderly patient and elaborate a poster and posters on the walls of the Unit to always keep them in mind in daily activities:

1. Respect for bodily intimacy: we will ask permission to undress, touch, explore... the body of the other person, explaining beforehand what is going to be done. With special delicacy at the moment of grooming.

2. Respect for the physical space: we will keep the box closed if the patient prefers, letting them know who is going to enter and for what purpose.

3. Gathering information from the person being attended: we will ask what is necessary, we are not entitled to ask everything.

4. Information among teammates involved in the care of the person: we will assess what information is transmitted to the team and what is reserved for oneself, if it does not contribute any benefit to the clinical process and may violate the patient's privacy.

5. Telephone information: we will not give clinical information over the telephone, except on exceptional occasions, with guarantees of knowing who we are talking to, with the patient's consent.

6. Respect for the privacy and participation of the family or related persons:

we will ask the patient who he/she wishes to be informed, since he/she has the right to choose with whom he/she wishes to share information about his/her state of health. The age of majority in health is 16 years old, and the patient has the right to information and confidentiality about himself/herself.

7. Custody of the clinical history: we will be attentive to the history, to avoid unwanted intrusion in the patient's privacy.

8. Privacy when receiving visitors during admission: patients have the right to choose who can visit them. We will try to improve the patient's image in the face of the visit, in order to reinforce their self-esteem.

9. Privacy when transferring patients to complementary tests or to other hospital wards: we will be careful, paying attention to physical exposure during transfers.

10. Respect for the patient's public and social image: no information is to be given to the media (accidents, assaults, public figures, etc.), unless there is express authorization.

\section{Measurement Indicators:}

Training:

a. Number of hours spent on humanization training. 
b. Number of hours spent on communication training.

Workshops: Number of workshops conducted.

Meetings: Number of hours spent in team meetings.

\section{Methodology}

The humanization plan is proposed as a set of activities aimed at professionals working in the Geriatrics Service of the Hospital Insular de Lanzarote. Discussion groups will be held among the professionals, every 15 days to assess and evaluate the activities that are developed in the different lines proposed.

\section{Conclusion}

Elderly users who are admitted to an Acute Geriatric Unit often spend a long period of time in it and even until the end of their days. Complex and stressful situations arise for both patients

\section{ISSN: 2574-1241}

DOI: $10.26717 /$ BJSTR.2021.35.005754

Ruben Octavio Luzardo Batista. Biomed J Sci \& Tech Res

(c) () This work is licensed under Creative Commons Attribution 4.0 License

Submission Link: https://biomedres.us/submit-manuscript.php and their relatives. That is why maintaining more humanized spaces that facilitate and create a welcoming feeling will improve these situations and their moods. It is important that patients do not perceive the Acute Geriatric Care Unit as a "strange and hostile environment". The training and awareness of the entire multidisciplinary team favors this process of humanization of healthcare institutions.

\section{References}

1. Eisenach JH, Sprung J, Clark M, Shanafelt TD, Johnson BD, et al. (2014) The psychological and physiological effects of acute occupational stress in new anesthesiology residents: a pilot trial. Anesthesiology 121(4): 878-893.

2. Fox M T, Persaud M, Maimets I, O’Brien K, Brooks D, et al. (2012) Effectiveness of acute geriatric unit care using acute care for elders components: A systematic review and meta-analysis. Journal of the American Geriatrics Society 60(12): 2237-2245.

3. RAE (n.d.). Real Academia Lengua Española.

$\begin{array}{ll}\text { BIOMEDICAL } & \text { Assets of Publishing with us } \\ \text { RESEARCHES } & \text { - Global archiving of articles } \\ \text { - Immediate, unrestricted online access }\end{array}$

